University of Wollongong

Research Online

Faculty of Engineering - Papers (Archive)

Faculty of Engineering and Information

Sciences

2011

\title{
Megavoltage cone beam CT near surface dose measurements: potential implications for breast radiotherapy
}

\author{
Alexandra Quinn \\ aq908@uowmail.edu.au \\ Lois C. Holloway \\ loish@uow.edu.au \\ Dean Cutajar \\ University of Wollongong, deanc@uow.edu.au \\ Nicholas Hardcastle \\ University of Wollongong, nhardc@uow.edu.au \\ Anatoly B. Rosenfeld \\ University of Wollongong, anatoly@uow.edu.au
}

See next page for additional authors

Follow this and additional works at: https://ro.uow.edu.au/engpapers

Part of the Engineering Commons

https://ro.uow.edu.au/engpapers/1707

\section{Recommended Citation}

Quinn, Alexandra; Holloway, Lois C.; Cutajar, Dean; Hardcastle, Nicholas; Rosenfeld, Anatoly B.; and Metcalfe, Peter E.: Megavoltage cone beam CT near surface dose measurements: potential implications for breast radiotherapy, Medical Physics: 38(11) 2011, 6222-6227.

https://ro.uow.edu.au/engpapers/1707

Research Online is the open access institutional repository for the University of Wollongong. For further information contact the UOW Library: research-pubs@uow.edu.au 
Authors

Alexandra Quinn, Lois C. Holloway, Dean Cutajar, Nicholas Hardcastle, Anatoly B. Rosenfeld, and Peter E. Metcalfe 


\title{
Megavoltage cone beam CT near surface dose measurements: potential implications for breast radiotherapy
}

\author{
Alexandra Quinn \\ Centre for Medical Radiation Physics, University of Wollongong, Wollongong, NSW 2522, Australia \\ and Liverpool Cancer Therapy Centre, Liverpool Hospital, Liverpool, NSW 2170, Australia \\ Lois Holloway \\ Centre for Medical Radiation Physics, University of Wollongong, Wollongong, NSW 2522, Australia; \\ Liverpool Cancer Therapy Centre, Liverpool Hospital, Liverpool, NSW 2170, Australia; and Department of \\ Human Oncology, University of Wisconsin-Madison, Wisconsin 53792 \\ Dean Cutajar \\ Centre for Medical Radiation Physics, University of Wollongong, Wollongong, NSW 2522, Australia \\ Nicholas Hardcastle \\ Centre for Medical Radiation Physics, University of Wollongong, Wollongong, NSW 2522, Australia and \\ Department of Human Oncology, University of Wisconsin-Madison, Wisconsin 53792 \\ Anatoly Rosenfeld \\ Centre for Medical Radiation Physics, University of Wollongong, Wollongong, NSW 2522, Australia \\ Peter Metcalfe \\ Centre for Medical Radiation Physics, University of Wollongong, Wollongong, NSW 2522, Australia and \\ Liverpool Cancer Therapy Centre, Liverpool Hospital, Liverpool, NSW 2170, Australia
}

(Received 30 March 2011; revised 8 August 2011; accepted for publication 31 August 2011; published 27 October 2011)

\begin{abstract}
Purpose: Cone beam computed tomography (CBCT) is fast becoming standard on modern linear accelerators. CBCT increases the dose to regions within and outside the treatment field, potentially increasing secondary cancer induction and toxicity. This study quantified megavoltage (MV) CBCT skin dose and compared it to skin dose delivered during standard tangential breast radiotherapy.

Method: Dosimetry was performed both in- and out-of-field using thermoluminescent dosimeters (TLDs) and a metal-oxide-semiconductor-field-effect-transistor (MOSFET) detector specifically designed for skin dosimetry; these were placed superficially on a female anthropomorphic phantom.

Results: The skin dose from a single treatment fraction ranged from 0.5 to $1.4 \mathrm{~Gy}$ on the ipsilateral breast, 0.031-0.18 Gy on the contralateral breast, and 0-0.02 Gy in the head and pelvic region. An 8 MU MV CBCT delivered a skin dose that ranged from 0.02 to $0.05 \mathrm{~Gy}$ in the chest region and was less than $0.01 \mathrm{~Gy}$ in the head and pelvis regions. One MV CBCT per fraction was found to increase the outof-field skin dose from both the CBCT and the treatment fields by approximately $20 \%$. The imaging dose as a percentage of treatment doses in the ipsilateral breast region was $3 \%$ for both dosimeters.

Conclusion: Imaging increases the skin dose to regions outside the treatment field particularly regions immediately adjacent the target volume. This small extra dose to the breasts should be considered when developing clinical protocols and assessing dose for clinical trials. (C) 2011 American Association of Physicists in Medicine. [DOI: 10.1118/1.3641867]
\end{abstract}

Key words: MOSFET, MV cone-beam CT, surface dosimetry, TLD

\section{INTRODUCTION}

Image-guided radiotherapy (IGRT) utilizes advanced imaging technology in the treatment room to ensure correct patient setup. Megavoltage (MV) cone beam computed tomography (CBCT) (Ref. 1) is a technology used for IGRT that employs the medical linear accelerator treatment beam and an amorphous silicon flat panel detector to acquire projections of the patient. Cone beam reconstruction technology is then used to reconstruct a volumetric image of the patient.

In an era of increasing use of IGRT technologies where multiple images are taken during the treatment course, extra radiation dose to the patient from imaging should be consid- ered. In particular, repeated imaging could potentially exacerbate radiation-induced skin toxicity by increasing the in-field skin dose. To the best of our knowledge, there have been no studies evaluating the influence of concomitant imaging on skin toxicity. In breast radiotherapy, total treatment doses of greater than 50 Gy and larger fraction sizes have been associated with greater skin toxicities. ${ }^{2-4}$ If imaging were to increase the fraction dose or total treatment dose, to $60 \mathrm{~Gy}$ for example, the patient may experience worse skin reactions. Radiationinduced skin toxicity is a side effect most breast cancer radiotherapy patients $(74 \%-100 \%)$ will experience during their treatment. $^{5-8}$ The dose to the skin can vary considerably inside and outside the treatment field. Knowledge of this dose is 
important in order to identify areas where unwanted skin reactions may occur. The imaging dose may also contribute to a delivered dose that exceeds the prescribed dose.

Skin dose can vary considerably due to its dependence upon a number of factors such as treatment field dimensions, the use of beam modifying devices, and the obliquity of the beam. Additionally, skin thickness varies considerably over the entire body; breast skin thickness ranges from 0.6 to $2.7 \mathrm{~mm} .{ }^{9,10}$ Consequently, skin dose is difficult to assess and measure, particularly in vivo. In vivo skin dose measurements can be acquired by measuring the dose at various depths and extrapolating to the desired depth. This technique can be completed with multiple thermoluminescent dosimeters (TLDs) of different thickness $^{11}$ or multilayer Gafchromic film. ${ }^{12}$ However, both methods can be time consuming due to multiple calibrations and both methods only provide retrospective dose information. Another option for in vivo skin dosimetry is to use a dosimeter with a small measurement depth (less than $1 \mathrm{~mm}$ ) such as carbon-loaded TLDs, ${ }^{13}$ radiochromic film, ${ }^{14}$ or metal-oxidesemiconductor-field-effect-transistor (MOSFETs). ${ }^{15}$ In the clinical environment, the most commonly used detectors for in vivo dosimetry are diodes and TLDs. ${ }^{16}$

The skin dose to various regions such as the contralateral breast from different breast radiotherapy techniques has been well reported. ${ }^{17-19}$ Kilovoltage $(\mathrm{kV})$ CBCT is the most common imaging modality provided by several vendors (XVI ${ }^{\circ}$, Elekta Oncology Systems; OBI, Varian Medical Systems; Artiste, Siemens); however, MV CBCT (Ref. 1) still makes up a substantial proportion of the imaging systems integrated with linear accelerators. In the literature, there is limited in vivo skin dose values available for MV CBCT, the image modality investigated in this study. A Monte Carlo study ${ }^{20}$ provides a single value for the whole body skin dose from a head and neck MV CBCT (0.59 cGy for an 8 MU MV CBCT) and a pelvis MV CBCT (0.94 cGy for an 8 MU MV CBCT). In this study, ${ }^{20}$ no region specific skin dose values were provided, such as the dose delivered in or out of the imaging field-ofview, and hence skin dose assessment to areas deemed at high risk of radiation skin damage, such as the eyes in head and neck radiotherapy, was not possible.

This report details a phantom study conducted for the purpose of investigating the additional skin dose a MV CBCT delivers to a female patient during a standard tangential breast radiotherapy fraction. Dosimetry was performed both in- and out-of-field using TLDs and a MOSFET detector specifically designed for skin dosimetry, the MOSkin MOSFET.

\section{MATERIALS AND METHODS}

\section{II.A. Phantom, treatment plan, and megavoltage cone beam CT}

A female anthropomorphic phantom (Radiology Support Devices, USA) was utilized for this study. Twenty-two point positions on the phantoms surface in the head and neck, chest, and pelvic region were chosen to measure the radiation skin dose from the breast radiotherapy treatment and MV CBCT image protocol described below. The measurement locations are shown in Fig. 1. The skin dose was measured with TLDs

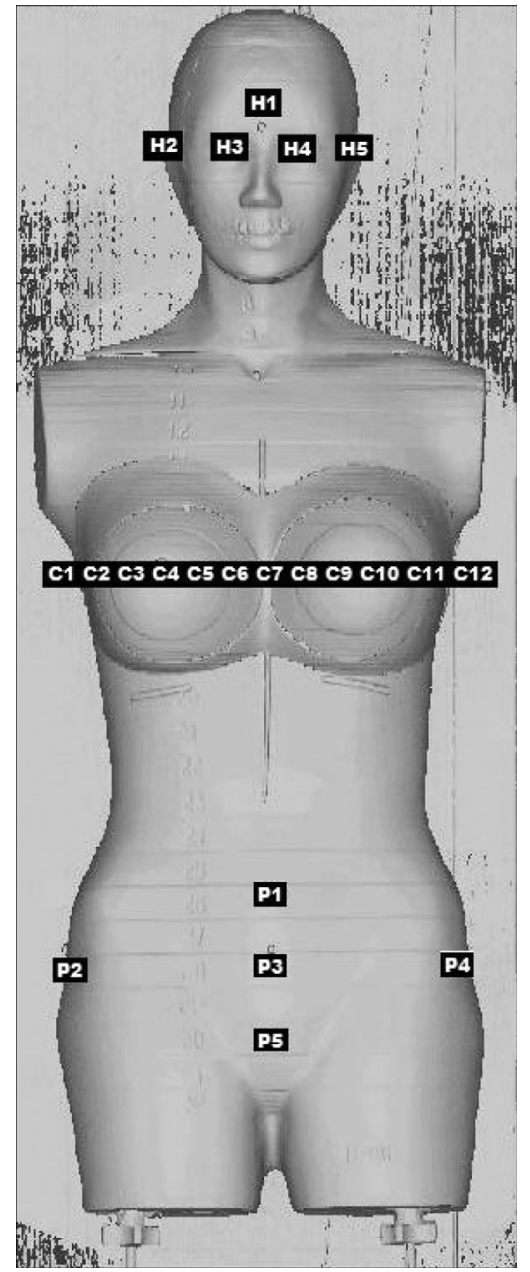

FIG. 1. Three-dimensional CT-render of female anthropomorphic phantom, illustrating the approximate radiation detector locations.

and MOSFETs, with specifications described in Secs. II B and II C, respectively.

A standard breast radiotherapy treatment plan was developed for the left breast according to departmental protocol. The plan consisted of opposed tangential breast fields, at gantry angles $309^{\circ}$ and $134^{\circ}$, with $15^{\circ}$ virtual wedges, and a standard dose regimen of $50 \mathrm{~Gy}$ (designed to be delivered in 2 Gy fractions). Five fractions were delivered to the phantom in a single measurement, this ensured detectors at outof-field treatment positions received a significant signal beyond the noise background threshold. Measured skin dose values were then extrapolated back to a single fraction dose.

A 60 MU MV CBCT imaging protocol was applied to the phantom. This high imaging dose assisted dosimetric response signal for both detectors and rendered background noise minimal. The image acquisition parameters included $6 \mathrm{MV}$ photons, $200^{\circ}$ rotation arc, $27.4 \times 27.4 \mathrm{~cm}$ fieldof-view, $256 \times 256$ reconstruction matrix, and $0.1 \mathrm{~cm}$ slice thickness. Measured dose values were scaled to Gy/MU and from this the dose for an $8 \mathrm{MU}$ protocol was determined. The contralateral breast, points $\mathrm{C} 1-\mathrm{C} 6$, and ipsilateral breast, points C7-C12, are within the MV CBCT field-of-view.

A daily imaging schedule was investigated in this study, as this would deliver the highest skin dose to the patient and 
thus a "worst case" scenario is presented. It is recognized that clinically for breast radiotherapy a weekly image schedule would be most likely. ${ }^{21,22}$

The breast treatment and MV CBCT protocol described above was delivered to the phantom using a Siemens Oncor linear accelerator (Siemens Medical Solutions, Erlangen, Germany) operating at $6 \mathrm{MV}$. Dose measurements were repeated twice to obtain statistical variation.

\section{II.B. Thermoluminescent dosimetry}

Standard $3 \times 3 \times 0.9 \mathrm{~mm}$ lithium-fluoride TLD-100 chips (Harshaw, Erlangen, Germany) with a measurement water equivalent depth (WED) of approximately $1 \mathrm{~mm}$ (Ref. 23) were used. TLD chips were irradiated to known doses within the expected range of $\mathrm{MV} \mathrm{CBCT}$ and breast radiotherapy energies (6 MV) and doses $(0.1,0.2,0.5,1$, and $2 \mathrm{~Gy})$ to establish TLD sensitivity and individual chip calibration factors. Only TLD chips with sensitivity ranging within $\pm 5 \%$ were selected for measurements in this study. TLD chips were read within $24 \mathrm{~h}$ of irradiation with a Rialto NE TLD reader (NE Technology Ltd, UK). Two TLD chips were placed at each location to reduce statistical error in the dose measurements.

\section{II.C. MOSFET dosimetry}

The MOSFET detector utilized for this study was the MOSkin, a MOSFET designed specifically for skin dosimetry. ${ }^{24}$ The MOSkin MOSFET has a uniform build-up layer with a measurement WED of $0.07 \mathrm{~mm},{ }^{24}$ the International Commission on Radiological Protection (ICRP) defines this depth as radiosensitive basal layer of the skin. ${ }^{25}$ The total detector size including packaging is $2 \times 5 \times 0.7 \mathrm{~mm}$. The MOSFETs surface dose response increases with increasing beam obliquity. ${ }^{19}$ Previous studies have utilized this MOSFET for in-field skin and surface dosimetry. ${ }^{19,26}$ Also for rectal wall interface dosimetry ${ }^{27}$ and for brachytherapy applications. $^{24}$

The accuracy of the MOSFET, utilized for this study, for out-of-field dosimetry was assessed. The MOSFET response for a $10 \times 10 \mathrm{~cm}, 6 \mathrm{MV}$ beam was measured at 2 and $15 \mathrm{~mm}$ depth $\left(\mathrm{d}_{\max }\right)$ in solid water, at the following distances from the central axis $0,6,8,10,15,20$, and $25 \mathrm{~cm}$. The $2 \mathrm{~mm}$ depth measurements were compared with an Attix ionization chamber (at $2 \mathrm{~mm}$ depth) in the exact same setup for comparison. The Attix chamber has a large guard ring and is optimized for build-up measurements. ${ }^{28}$ The MOSFET dose measurements were then compared with a thimble chamber (at $15 \mathrm{~mm}$ depth) as the thimble chamber is not optimized for measurements in the build-up region.

MOSFET surface doses at each measurement location were obtained by multiplying the difference of the threshold voltage before and after irradiation by a calibration factor. The calibration factor was determined by irradiating the MOSFETs to a known dose in standard conditions in a $6 \mathrm{MV}$ beam, before and after measurements. The average calibration factor was utilized for treatment and imaging measurements.

\section{RESULTS}

The out-of-field MOSFET response is illustrated in Fig. 2 for a $6 \mathrm{MV}$ beam $10 \times 10 \mathrm{~cm}$ field at distances $0,6,8,10$, 15,20 , and $25 \mathrm{~cm}$ from the central axis, and at depths 2 and $15 \mathrm{~mm}$. Agreement within $0.1 \%$ was observed between the MOSFET and ionization chamber measurements, with the exception at depth $2 \mathrm{~mm}, 6 \mathrm{~cm}$ from the central axis, where $2 \%$ agreement was observed. Discrepancy between the Attix ionization chamber and the MOSFETs at a distance $6 \mathrm{~cm}$ from the central axis is attributable to the larger surface area of the chamber resulting in a section of the attix chamber sensitive volume residing in the radiation field.

The skin dose measured for a single treatment fraction and an $8 \mathrm{MU}$ MV CBCT scan in the head, contralateral breast, ipsilateral breast and pelvic region is outlined in Table I. The skin dose from a single 8 MU MV CBCT was less than $0.01 \mathrm{~Gy}$ in the head and pelvis regions and ranged from 0.02 to $0.05 \mathrm{~Gy}$ in the chest region. The skin dose from a single treatment fraction was less than 0.02 Gy in the head and pelvic region and ranged from 0.5 to 1.4 Gy on the ipsilateral breast and $0.031-0.18$ Gy on the contralateral breast.

Utilizing the measured values from Table I, the single 8 MU MV CBCT image skin dose is presented as a percentage of a single treatment fraction skin dose in Fig. 3. The imaging dose as a percentage of treatment dose is highest for the most lateral point of the contralateral breast.

\section{DISCUSSION}

Skin dose measurements at a range of locations both in and out of a tangential breast treatment area for MV CBCT and treatment delivery have been presented. The MOSkin MOSFET was shown to be accurate for out-of-field dosimetry, with the MOSFET and ionization chamber measured out-of-field doses in agreement to within $0.1 \%$ (with the exception of one point) of the maximal dose in the beam. This agreed with a similar study ${ }^{29}$ investigating the MOSFET peripheral dose accuracy. At $15 \mathrm{~mm}$ depth, $200 \mathrm{~mm}$

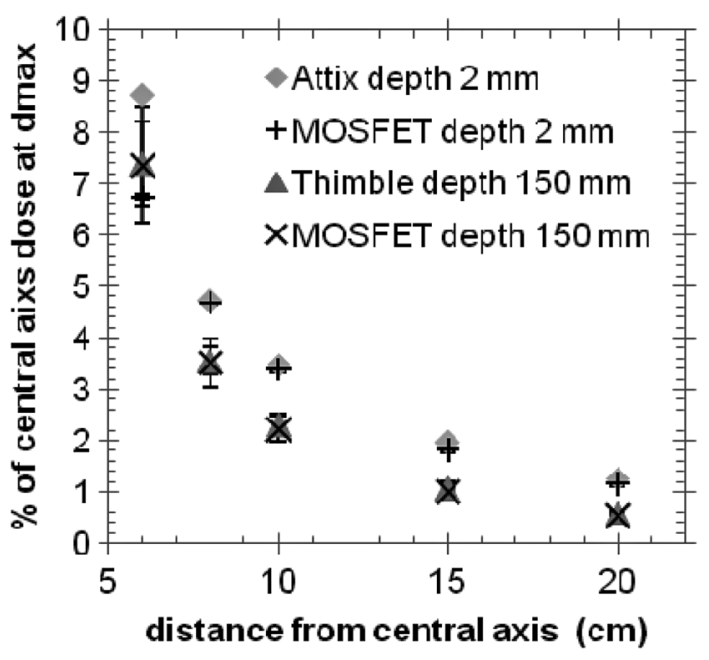

FIG. 2. MOSFET response measured out-of-field for a $6 \mathrm{MV} 10 \times 10 \mathrm{~cm}$ photon field. The error bars represent the $95 \%$ confidence interval of the mean of three measurements. 
from the central axis, $4000 \mathrm{MU}$ was delivered to the centre of the $10 \times 10 \mathrm{~cm}$ field to ensure the MOSFETs received an adequate dose whereas $100 \mathrm{MU}$ was delivered for the thimble chamber. However, the dose delivered to the MOSFETs out-of-field was significantly increased in comparison to the ionization chambers, to ensure sufficient signal to noise ratio. While using extra MU was practical in this phantom study, using MOSFETs with a higher sensitivity would be advantageous for patient dosimetry. The current study used the MOSkin MOSFETs at $+12 \mathrm{~V}$ bias giving a sensitivity of $2.5 \mathrm{mV} \mathrm{cGy}^{-1}$, there is potential to significantly increase the sensitivity by increasing the gate oxide thickness ${ }^{30,31}$ and gate bias. $^{32}$

The present study utilized two detectors to measure skin dose, both detectors illustrated that MV CBCT verification imaging increases the skin dose to areas that would not receive a significant skin dose from treatment alone. If $\mathrm{MV}$ CBCT was utilized for daily image verification during a standard tangential breast radiotherapy treatment fraction the skin dose at the lateral side of the contralateral breast would approximately double. This is attributable to the contralateral breast being within the MV CBCT field-of-view, as the MV CBCT acquires projection images in a $200^{\circ}$ arc around the patient to reconstruct the $3 \mathrm{D}$ image. Reduced dose may be acquired by reducing the arc of imaging, however, image quality would be reduced due to the reduced projection angle.

The skin dose from an $8 \mathrm{MU}$ MV CBCT ranged from 0.03 to $0.05 \mathrm{~Gy}$ and 0.02 to $0.03 \mathrm{~Gy}$ in the chest region for the TLDs and MOSFETs, respectively. In comparison, within the imaging field of a $\mathrm{kV}$ CBCT acquired for breast radiotherapy setup a previous study found the breast skin dose, measured with the same type of TLDs, received a lower dose of $0.01 \mathrm{~Gy}^{33}$ Limited organ and tissue dose measurements from a chest MV CBCT have been investigated previously with treatment planning systems. ${ }^{34,35}$ These studies did not provide skin dose values; thus, a comparison with the present data could not be made.

The skin dose from a single 2 Gy treatment fraction ranged from 0.5 to $1.4 \mathrm{~Gy}$ on the ipsilateral breast, $0.031-0.18$ Gy on the contralateral breast, and $0-0.02$ Gy in the head and pelvic region. The contralateral breast skin dose values are in agreement with a similar study which reported doses of $0.022-0.13 \mathrm{~Gy} .{ }^{18}$ The higher doses measured in the present study are attributable to the phantom breasts utilized in this study not settling laterally as patients breasts tend to do. For this reason, the phantoms contralateral breast remains closer to the beam edge and hence may receive a higher dose.

The MOSFET uncertainty was large at positions outside the CBCT field-of-view and out-of-field of the treatment beams, see Table I. This is attributable to the MOSFETs lower detection limit of approximately $0.15 \mathrm{cGy} .{ }^{36}$ For this reason, the MOSFETs utilized in this study would not be suitable for clinical skin dose measurements far from the treatment or imaging field-of-views unless, as mentioned above, the gate oxide thickness ${ }^{30,31}$ and gate bias ${ }^{32}$ is increased.

To the best of our knowledge, there have been no studies evaluating the influence of concomitant imaging on skin

TABLE I. Skin dose measured with TLDs and MOSkins from a single treatment fraction and a single 8 MU MV CBCT to the head and neck region (H1-H6), the contralateral breast (lateral C1-medial C6), the ipsilateral breast (medial C7-lateral C12), and the pelvic region (P1-P5). The detector positions are detailed in Fig. 1.

\begin{tabular}{|c|c|c|c|c|}
\hline \multirow[b]{2}{*}{ Position } & \multicolumn{2}{|c|}{ Single treatment fraction } & \multicolumn{2}{|c|}{ Single 8 MU MV CBCT } \\
\hline & $\begin{array}{c}\text { TLD } \\
\text { Dose (cGy) } \pm 95 \% \text { CI }\end{array}$ & $\begin{array}{c}\text { MOSFET } \\
\text { Dose }(\mathrm{cGy}) \pm 95 \% \mathrm{CI}\end{array}$ & $\begin{array}{c}\text { TLD } \\
\text { Dose (cGy) } \pm 95 \% \text { CI }\end{array}$ & $\begin{array}{c}\text { MOSFET } \\
\text { Dose (cGy) } \pm 95 \% \text { CI }\end{array}$ \\
\hline H1 & $0.69 \pm 0.01$ & $0.57 \pm 0.34$ & $0.09 \pm 0.05$ & $0.02 \pm 0.23$ \\
\hline $\mathrm{H} 2$ & $1.37 \pm 0.11$ & $1.67 \pm 0.13$ & $0.20 \pm 0.02$ & $0.25 \pm 0.29$ \\
\hline H3 & $1.79 \pm 0.12$ & $2.36 \pm 0.56$ & $0.24 \pm 0.08$ & $0.16 \pm 0.20$ \\
\hline H4 & $1.82 \pm 0.11$ & $1.94 \pm 0.41$ & $0.22 \pm 0.01$ & $0.19 \pm 0.53$ \\
\hline H5 & $0.89 \pm 0.03$ & $0.94 \pm 0.45$ & $0.20 \pm 0.04$ & $0.21 \pm 0.14$ \\
\hline $\mathrm{C} 1$ & $3.10 \pm 0.11$ & $3.47 \pm 0.67$ & $3.68 \pm 0.39$ & $2.19 \pm 0.27$ \\
\hline $\mathrm{C} 2$ & $4.19 \pm 0.13$ & $4.43 \pm 0.42$ & $3.57 \pm 0.45$ & $2.10 \pm 0.35$ \\
\hline $\mathrm{C} 3$ & $7.40 \pm 0.22$ & $7.82 \pm 0.51$ & $2.97 \pm 0.13$ & $1.90 \pm 0.17$ \\
\hline $\mathrm{C} 4$ & $12.7 \pm 0.09$ & $12.8 \pm 0.59$ & $3.36 \pm 0.45$ & $2.08 \pm 0.22$ \\
\hline $\mathrm{C} 5$ & $17.9 \pm 0.48$ & $18.0 \pm 0.63$ & $3.80 \pm 0.52$ & $2.43 \pm 0.24$ \\
\hline C6 & $24.2 \pm 2.69$ & $26.7 \pm 1.37$ & $4.29 \pm 0.73$ & $2.77 \pm 0.22$ \\
\hline $\mathrm{C} 7$ & $57.8 \pm 3.22$ & $48.8 \pm 1.43$ & $4.47 \pm 0.17$ & $2.99 \pm 0.26$ \\
\hline $\mathrm{C} 8$ & $132 \pm 2.32$ & $88.1 \pm 3.30$ & $3.25 \pm 0.27$ & $2.16 \pm 0.25$ \\
\hline C9 & $130 \pm 5.25$ & $99.5 \pm 3.25$ & $2.90 \pm 0.06$ & $1.84 \pm 0.08$ \\
\hline $\mathrm{C} 10$ & $151 \pm 1.27$ & $120 \pm 1.44$ & $2.71 \pm 0.31$ & $1.83 \pm 0.09$ \\
\hline $\mathrm{C} 11$ & $128 \pm 4.21$ & $103 \pm 2.64$ & $3.17 \pm 0.19$ & $2.25 \pm 0.23$ \\
\hline $\mathrm{C} 12$ & $135 \pm 2.86$ & $96.8 \pm 2.66$ & $3.91 \pm 0.37$ & $2.74 \pm 0.14$ \\
\hline P1 & $2.25 \pm 0.01$ & $2.63 \pm 0.26$ & $0.62 \pm 0.07$ & $0.66 \pm 0.26$ \\
\hline $\mathrm{P} 2$ & $0.60 \pm 0.04$ & $1.10 \pm 0.09$ & $0.22 \pm 0.10$ & $0.20 \pm 0.85$ \\
\hline P3 & $1.13 \pm 0.01$ & $1.24 \pm 0.32$ & $0.28 \pm 0.10$ & $0.29 \pm 0.50$ \\
\hline P4 & $1.37 \pm 0.25$ & $1.31 \pm 0.15$ & $0.23 \pm 0.01$ & $0.24 \pm 0.51$ \\
\hline P5 & $0.51 \pm 0.00$ & $0.36 \pm 0.23$ & $0.12 \pm 0.07$ & $0.20 \pm 0.86$ \\
\hline
\end{tabular}


toxicity. The impact of the MV CBCT imaging dose on skin toxicity was assessed according to studies which have investigated the deterministic and stochastic effects of skin irradiation. $^{2,37}$ This is not ideal, however, was deemed reasonable. Daily imaging was considered in this study, as this imaging schedule would deliver the highest imaging dose to the patient. This schedule would most likely not increase the possibility of contralateral breast skin reactions as it would not raise the total contralateral breast skin dose above the single fraction dose threshold (5 Gy) or total fractionated dose threshold (approximately $20 \mathrm{~Gy}$ ) for mild deterministic effects. ${ }^{2}$ However, the dose delivered to the contralateral breast during radiotherapy treatment is associated with an increased long-term risk of contralateral breast cancer, ${ }^{38}$ the additional imaging dose may increase this risk. In the ipsilateral breast region (points $\mathrm{C} 7-\mathrm{C} 12$ ) the imaging dose as a percentage of treatment dose was as expected minimal (up to 3\%), see Fig. 3. This is attributable to these measurement points being within both the treatment and CBCT field-ofview and the imaging dose being small in comparison to the treatment dose, see Table I. The imaging dose to the head and pelvic regions was up to $20 \%$ of the out-of-field skin dose delivered from the treatment at the same locations.

The contralateral breast (points C1-C6), head (points $\mathrm{H} 1-\mathrm{H} 5)$ and pelvis (P1-P5) skin dose from the treatment alone consists of leakage, extra focal scatter and electron contamination. The contralateral breast is in the MV CBCT field-of-view and out-of-field of the treatment beams. Hence, the magnitude of the MV CBCT dose contribution to the total contralateral breast dose is significantly higher than that of the breast radiotherapy treatment. The delivered dose from an $8 \mathrm{MU}$ MV CBCT is up to 118 and $62 \%$ of the dose delivered from treatment, for the TLDs and MOSFETs, respectively. An $8 \mathrm{MU}$ MV CBCT increases the dose delivered to location $\mathrm{C} 1$ from $0.03 \pm 0.01 \mathrm{~Gy}$, for both detectors, for a single treatment fraction to $0.07 \pm 0.02 \mathrm{~Gy}$ and $0.05 \pm 0.02 \mathrm{~Gy}$, for TLDs and MOSFETs, respectively. The large percentage difference between the TLD and MOSFETs is attributable to the detectors measuring similar skin dose values out-of-field of the treatment beams (at point $\mathrm{C} 1$ a difference of $0.5 \mathrm{cGy}$ ) and different skin dose values in the field-of-view of the CBCT scan (at point C1, a difference of 1.5 cGy). The dose discrepancy in-field of both the MV

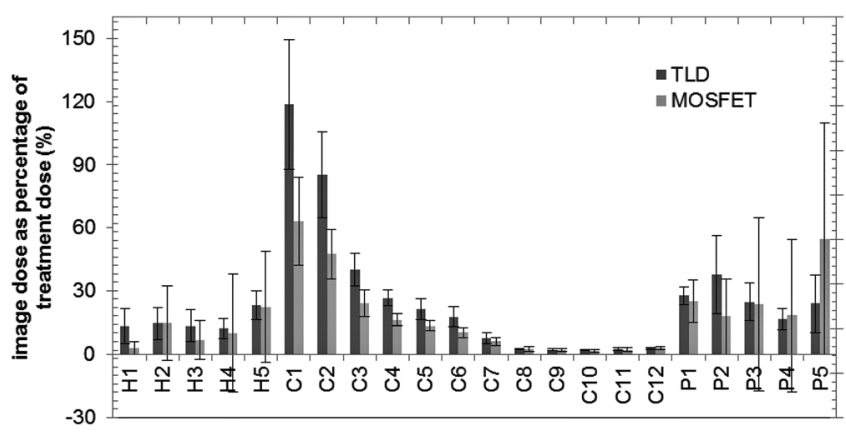

FIG. 3. The measured skin dose from a single $8 \mathrm{MU}$ MV CBCT is presented as a percentage of the measured skin dose from a single treatment fraction. The error bars represent $95 \%$ confidence intervals.
CBCT and treatment beams is attributable to differences in effective depth dose measurements. The MOSkin MOSFET WED of measurement is $0.07 \mathrm{~mm}$ whereas for the TLDs the WED is approximately $1 \mathrm{~mm}$. Large charged-particle disequilibrium exists at these depths and thus a small change in depth will result in a large change in dose measured. Out-offield the photon spectrum does not vary significantly ${ }^{39,40}$ and hence the measurement WED of the detector has little effect on the dose measurement. Consequently, the TLD and MOSFET skin dose measurements in the head and pelvic region were within one standard deviation of each other.

Measurements in this study were completed under ideal conditions in that the phantom utilized was moderately inflexible and hence could be set up consistently. The single size and shape of the female anthropomorphic phantom limited the study as the interpatient variation seen in practice is not represented by these measurements. Additionally, the phantoms breasts sit upright on the chest wall and do not fall laterally with gravity, as a patient's breast might. Consequently, the phantoms contralateral breast remains closer to the edge of the treatment beam and hence may result in a higher measured dose in comparison to a typical patient.

Knowledge of the imaging skin dose separate from that of the treatment dose is necessary in order to assess its impact on side effects such as skin toxicity. This can be accomplished with the TLDs and MOSFETs utilized in this study. The MOSFETs allow real time skin dose measurements and hence may be more practical for future in-field in vivo measurements.

\section{CONCLUSION}

One MV CBCT per fraction was found to increase the out-of-field skin dose by approximately $20 \%$. The contralateral breast skin dose from the treatment alone consists of leakage, extra focal scatter and electron contamination. The magnitude of the MV CBCT dose contribution is significantly higher than this. It is up to 118 and $62 \%$ of the dose delivered from treatment, for the TLDs and MOSFETs, respectively. In comparison, the imaging dose as a percentage of treatment doses in the ipsilateral breast region was $3 \%$ for both dosimeters. This extra dose will most likely not increase the skin toxicity in the treated breast. The skin dose to the contralateral breast is probably not enough to induce skin reactions but will add to the risk of second malignancy. ${ }^{38}$ With the advent of new interface dosimeters accurate in vivo skin dose assessment is possible, linking this dose assessment to patient's reactions and secondary cancer risk remains the next challenge.

\section{ACKNOWLEDGMENTS}

The authors wish to thank the Illawarra Cancer Therapy Centre for lending their anthropomorphic phantom. The author A. Q. wishes to acknowledge Liverpool and Macarthur Cancer Therapy Centres trust funds for scholarship. The author P. M. wishes to acknowledge financial assistance from the NSW Cancer Institute Clinical Leaders program. 
${ }^{1}$ O. Morin, A. Gillis, J. Chen, M. Aubin, K. Bucci, M. Roach, and J. Pouliot, "Megavoltage cone-beam CT: system description and clinical applications," Med. Dosim. 31, 51-61 (2006).

${ }^{2}$ J. O. Archambeau, R. Pezner, and T. Wasserman, "Pathophysiology of irradiated skin and breast," Int. J. Radiat. Oncol., Biol., Phys. 31, 1171-1185 (1995).

${ }^{3}$ R. Gorodetsky, C. Lotan, K. Piggot, L. J. Pierce, I. Polyansky, S. Dische, M. I. Saunders, A. S. Lichter, and A. Vexler, "Late effects of dose fractionation on the mechanical properties of breast skin following postlumpectomy radiotherapy," Int. J. Radiat. Oncol., Biol., Phys. 45, 893-900 (1999).

${ }^{4}$ M. E. Taylor, C. A. Perez, K. J. Halverson, R. R. Kuske, G. W. Philpott, D. M. Garcia, J. E. Mortimer, R. J. Myerson, D. Radford, and C. Rush, "Factors influencing cosmetic results after conservation therapy for breast cancer," Int. J. Radiat. Oncol., Biol., Phys. 31, 753-764 (1995).

${ }^{5}$ E. Berthelet, P. T. Truong, K. Musso, V. Grant, W. Kwan, V. Moravan, K. Patterson, and I. A. Olivotto, "Preliminary reliability and validity testing of a new skin toxicity assessment tool (STAT) in breast cancer patients undergoing radiotherapy," Am. J. Clin. Oncol. 27, 626-631 (2004).

${ }^{6}$ G. M. Freedman, T. Li, N. Nicolaou, Y. Chen, C. C. M. Ma, and P. R. Anderson, "Breast intensity-modulated radiation therapy reduces time spent with acute dermatitis for women of all breast sizes during radiation," Int. J. Radiat. Oncol., Biol., Phys. 74, 689-694 (2009).

${ }^{7}$ M. T. Knobf and Y. Sun, "A longitudinal study of symptoms and self-care activities in women treated with primary radiotherapy for breast cancer," Cancer Nurs. 28, 210-218 (2005).

${ }^{8}$ Y. Wengström, C. Häggmark, H. Strander, and C. Forsberg, "Perceived symptoms and quality of life in women with breast cancer receiving radiation therapy," Eur. J. Oncol. Nurs. 4, 78-88 (2000).

${ }^{9}$ T. L. Pope, M. E. Read, T. Medsker, A. J. Buschi, and A. N. Brenbridge, "Breast skin thickness: normal range and causes of thickening shown on film-screen mammography," J. Can. Assoc. Radiol. 35, 365-368 (1984).

${ }^{10}$ S.-Y. Huang, J. M. Boone, K. Yang, A. L. C. Kwan, and N. J. Packard, "The effect of skin thickness determined using breast CT on mammographic dosimetry," Med. Phys. 35, 1199-1206 (2008).

${ }^{11}$ T. Kron, M. Butson, F. Hunt, and J. Denham, "TLD extrapolation for skin dose determination in vivo," Radiother. Oncol. 41, 119-123 (1996).

${ }^{12}$ T. Cheung, M. J. Butson, and P. K. N. Yu, "Multilayer gafchromic film detectors for breast skin dose determination in vivo," Phys. Med. Biol. 47, N31-N37 (2002).

${ }^{13}$ P. M. Ostwald, T. Kron, C. S. Hamilton, and J. W. Denham, "Clinical use of carbon-loaded thermoluminescent dosimeters for skin dose determination,” Int. J. Radiat. Oncol., Biol., Phys. 33, $943-950$ (1995).

${ }^{14}$ S. Devic, J. Seuntjens, W. Abdel-Rahman, M. Evans, M. Olivares, E. B. Podgorsak, T. Vuong, and C. G. Soares, "Accurate skin dose measurements using radiochromic film in clinical applications," Med. Phys. 33, 1116-1124 (2006)

${ }^{15}$ M. J. Butson, A. Rozenfeld, J. N. Mathur, M. Carolan, T. P. Y. Wong, and P. E. Metcalfe, "A new radiotherapy surface dose detector: The MOSFET," Med. Phys. 23, 655-658 (1996).

${ }^{16} \mathrm{M}$. Essers and B. J. Mijnheer, "In vivo dosimetry during external photon beam radiotherapy," Int. J. Radiat. Oncol., Biol., Phys. 43, 245-259 (1999).

${ }^{17}$ T. C. S. Woo, J. P. Pignol, E. Rakovitch, T. Vu, D. Hicks, P. O’Brien, and K. Pritchard, "Body radiation exposure in breast cancer radiotherapy: Impact of breast IMRT and virtual wedge compensation techniques," Int. J. Radiat. Oncol., Biol., Phys. 65, 52-58 (2006).

${ }^{18} \mathrm{~J}$. Burmeister, N. Alvarado, S. Way, P. McDermott, T. Bossenberger, H. Jaenisch, R. Patel, and T. Washington, "Assessment and minimization of contralateral breast dose for conventional and intensity modulated breast radiotherapy," Med. Dosim. 33, 6-13 (2008).

${ }^{19}$ A. Kelly, N. Hardcastle, P. Metcalfe, D. Cutajar, A. Quinn, K. Foo, M. Cardoso, S. Barlin, and A. B. Rosenfeld, "Surface dosimetry for breast radiotherapy in the presence of immobilization cast material," Phys. Med. Biol. 56, 1001-1013 (2011).

${ }^{20}$ J. Gu, B. Bednarz, X. G. Xu, and S. B. Jiang, "Assessment of patient organ doses and effective doses using the VIP-Man adult male phantom for selscted cone-beam CT imaging procedures during image guided radiation therapy," Radiat. Prot. Dosim. 131, 431-443 (2008).

${ }^{21}$ R. Topolnjak, J.-J. Sonke, J. Nijkamp, C. Rasch, D. Minkema, P. Remeijer, and C. van Vliet-Vroegindeweij, "Breast patient setup error assessment: comparison of electronic portal image devices and cone-beam computed tomography matching results," Int. J. Radiat. Oncol., Biol., Phys. 78, 1235-1243 (2010).

${ }^{22}$ A. van Mourik, S. van Kranen, S. den Hollander, J. J. Sonke, M. van Herk, and C. van Vliet-Vroegindeweij, "Effects of setup errors and shapes changes on breast radiotherapy," Int. J. Radiat. Oncol., Biol., Phys. 79 1557-1564 (2011)

${ }^{23}$ T. Kron, A. Elliot, T. Wong, G. Showell, B. Clubb, and P. Metcalfe, "Xray surface dose measurements using TLD extrapolation," Med. Phys. 20, 703-711 (1993).

${ }^{24}$ I. S. Kwan, A. B. Rosenfeld, Z. Y. Qi, D. Wilkinson, M. L. F. Lerch, D. L. Cutajar, M. Safavi-Naeni, M. Butson, J. A. Bucci, Y. Chin, and V. L. Perevertaylo, "Skin dosimetry with new MOSFET detectors," Radiat. Meas. 43, 929-932 (2008).

${ }^{25}$ ICRP, The Biological Basis for Dose Limitation in the Skin (ICRP Publication, Pergamon, Oxford, 1992), Vol. 59.

${ }^{26}$ N. Hardcastle, E. Soisson, P. Metcalfe, A. B. Rosenfeld, and W. A. Tome, "Dosimetric verification of helical tomotherapy for total scalp irradiation," Med. Phys. 35, 5061-5068 (2008).

${ }^{27}$ N. Hardcastle, D. L. Cutajar, P. E. Metcalfe, M. L. F. Lerch, V. L. Perevertaylo, W. A. Tomé, and A. B. Rosenfeld, "In vivo real-time rectal wall dosimetry for prostate radiotherapy," Phys. Med. Biol. 55, 3859-3871 (2010)

${ }^{28} \mathrm{~F}$. Attix, "Application of a commercial solid-water plane-parallel ionization chamber to the AAPM TG-21 protocol," Med. Phys. 20, 735-737 (1993).

${ }^{29}$ M. J. Butson, T. Cheung, and P. K. N. Yu, "Peripheral dose measurement with a MOSFET detector," Appl. Radiat. Isot. 62, 631-634 (2005).

${ }^{30}$ A. B. Rosenfeld, "MOSFET dosimetry on modern radiation oncology modalities," Radiat. Prot. Dosim. 101, 393-398 (2002)

${ }^{31}$ G. Ensell, A. Holmes-Siedle, and L. Adams, "Thick oxide pMOSFET dosimeters for high energy radiation,” Nucl. Instrum. Methods Phys. Res. A 269, 655-658 (1988)

${ }^{32}$ P. Litovchenko, L. Barabash, A. Rosenfeld, V. Khivrich, O. Zinets, V. Kuts, I. Marusan, V. Petrov, G. Sluchenkov, and G. Koval, "MOS structure for emergency gamma and proton dosimetry," Radiat. Prot. Dosim. 33, 179 (1990)

${ }^{33}$ C. A. McBain, A. M. Henry, J. Sykes, A. Amer, T. Marchant, C. M Moore, J. Davies, J. Stratford, C. McCarthy, B. Porritt, P. Williams, V. S Khoo, and P. Price, "X-ray volumetric imaging in image-guided radiotherapy: The new standard in on-treatment imaging," Int. J. Radiat. Oncol., Biol., Phys. 64, 625-634 (2006).

${ }^{34}$ L. C. Peng, C. C. J. Yang, S. Sim, M. Weiss, and A. Bielajew, "Dose comparison of megavoltage cone-beam and orthogonal-pair portal images," J. Appl. Clin. Med. Phys. 8, 10-20 (2007).

${ }^{35}$ A. E. VanAntwerp, S. M. Raymond, M. C. Addington, S. Gajdos, A. Vassil, and P. Xia, "Dosimetric evaluation between megavoltage cone-beam computed tomography and body mass index for intracranial, thoracic, and pelvic localization," Med. Dosim. 36, 284-291 (2011).

${ }^{36} \mathrm{D}$. Peet and M. Pryor, "Evaluation of a MOSFET radiation sensor for the measurement of entrance surface dose in diagnostic radiology," Br. J. Radiol. 72, 562-568 (1999).

${ }^{37}$ L. K. Wagner, P. J. Eifel, and R. A. Gelse, "Potential biological effects following high x-ray dose interventional procedures," J. Vasc. Interv. Radiol. 5, 71-84 (1994).

${ }^{38} \mathrm{X}$. Gao, S. G. Fisher, and B. Emami, "Risk of second primary cancer in the contralateral breast in women treated for early-stage breast cancer: A population-based study,” Int. J. Radiat. Oncol., Biol., Phys. 56, 1038-1045 (2003).

${ }^{39}$ S. F. Kry, U. Titt, F. Ponisch, D. Followill, O. N. Vassiliev, R. A. White, R. Mohan, and M. Salehpour, "A monte carlo model for calculating outof-field dose from a Varian 6 MV beam," Med. Phys. 33, 4405-4413 (2006)

${ }^{40} \mathrm{C}$. R. Edwards and P. J. Mountford, "Near surface photon energy spectra outside a 6 MV field edge," Phys. Med. Biol. 49, N293-N301 (2004). 